\section{A regulação assistencial e a produção do cuidado: um arranjo potente para qualificar a atenção}

\author{
Healthcare regulation and healthcare management \\ as tools to assure comprehensiveness and \\ equity in health
}

Rossana Staevie Baduy 1

Laura Camargo Macruz Feuerwerker 2

Marlene Zucoli 3

Joelma Teixeira Borian 3
1 Centro de Ciências da Saúde, Universidade Estadual de Londrina, Londrina, Brasil.

2 Faculdade de Saúde Pública, Universidade de São Paulo, São Paulo, Brasil. 3 Secretaria Municipal de Saúde, Prefeitura Municipal de Londrina, Londrina, Brasil.

Correspondência R. S. Baduy

Departamento de Saúde Coletiva, Centro de Ciências da Saúde, Universidade Estadual de Londrina.

Rua Robert Koch 60, Londrina, PR 86038-350, Brasil. robaduy@gmail.com

\begin{abstract}
Healthcare production that assures comprehensiveness and equity has posed a challenge for Brazil's Unified National Health System. The aim of this study was to contribute to the debate on the issue, analyzing an experience with municipal healthcare management using regulation to improve quality of care. We conducted a case study in which data were obtained through direct observation, interviews, written narratives, and document analysis. The research subjects were health services managers and health workers. The results feature the concept of a living network, produced actively by building relations between the health workers in the different services. The study highlights the measures to support the care produced by the teams, based on healthcare regulation and work processes management. The results were expressive in upgrading the care, reducing the waiting lines for the specialties, and increasing the health workers'satisfaction. The key feature in the process was the evident need to enrich (through acts) the technical knowledge with light technologies and active movements by management in the production of care.
\end{abstract}

Health Management; Comprehensive Health Care; Family Health

\section{Introdução}

A gestão dos sistemas e serviços de saúde no Brasil é um grande desafio para a consolidação do Sistema Único de Saúde (SUS). Houve uma ampliação das ações e dos serviços ofertados, sobretudo pelos municípios, porém, ainda estamos distantes da rede contínua de cuidados integrais, indispensável para integrar as diferentes etapas de atenção, otimizar a aplicação dos recursos do SUS e consolidar sua legitimidade junto aos usuários 1,2 .

Além disso, a mudança do perfil demográfico e epidemiológico da população, destacando-se o envelhecimento e a alta prevalência dos agravos e doenças não transmissíveis, amplia os desafios para os sistemas de saúde. Esses agravos requerem convivência prolongada dos usuários com os profissionais de saúde e reforçam a necessidade de projetos cuidadores que favoreçam a manutenção e ampliação da sua autonomia. Para tanto, além de o usuário ocupar um novo papel na produção da saúde, são indispensáveis arranjos que propiciem a continuidade da atenção e que favoreçam a integralidade do cuidado. Também a articulação entre os serviços dos diversos níveis de atenção é um desafio importante, agravado pelo fato de estarem organizados e financiados segundo lógicas distintas. Isso implica limitada resolubilidade, descontinuidade, desresponsabilização, retrabalho e perda de qualidade da atenção prestada ao usuário 3,4,5. 
A literatura revela não serem esses problemas exclusivos do sistema de saúde brasileiro. Repetem-se em todos os países que perseguem a integralidade na atenção à saúde de seus cidadãos, como os países da União Européia. Estudos apontam o aumento das condições crônicas como o maior desafio a ser enfrentado, no século XXI, pelos sistemas de saúde preocupados com a equidade, eficiência e efetividade. Apontam também a fragmentação dos serviços como uma dificuldade para a produção da integralidade, mesmo naqueles países onde há uma forte atenção primária e serviços de atenção domiciliar. Alguns autores destacam a necessidade de estudar novos arranjos em experimentação para o enfrentamento dos problemas crônicos de saúde nas populações $4,6,7,8$.

Já Iriart et al. 9 indicam ainda que as reformas dos sistemas de saúde nos anos 90 abordavam os problemas de uma perspectiva economicista, reduzindo os problemas às questões financeiras, de escassez ou má distribuição de recursos. Desse modo deixavam de lado as pessoas e seus problemas, colocando no centro do debate as soluções vinculadas à eficiência e ao custo benefício. É nessa lógica que muitas vezes se opera a regulação.

No Brasil, a regulação tem sido discutida endereçando a fragmentação do cuidado e a dificuldade de acesso a serviços e procedimentos. $\mathrm{O}$ debate mais aprofundado em relação ao conceito, práticas e finalidades da regulação, controle, avaliação e auditoria em saúde iniciou-se a partir de 2001-2002 com as Normas Operacionais de Assistência à Saúde 01/2001 e 01/2002. O Pacto pela Saúde 200610 também traz a idéia da regulação, do matriciamento e das linhas de cuidado como alternativas para articular gestão do sistema e produção do cuidado.

Conhecer as experiências que vêm sendo desenvolvidas na construção de arranjos inovadores para o enfrentamento desses desafios é crucial para o SUS neste momento.

\section{O percurso metodológico}

Este artigo é fruto de uma pesquisa de doutorado em que foi estudada a produção da integralidade no SUS de Londrina, Paraná. O estudo analisou o espaço da micropolítica e as ferramentas tecnopolíticas mobilizadas pelos diferentes atores institucionais para a gestão da produção do cuidado em saúde, utilizando uma abordagem qualitativa. Realizou-se um estudo de caso, com as seguintes estratégias de aproximação ao campo: observação, entrevistas semiestruturadas, narrativas escritas e análise documental. Foi utilizado o diário de campo para o registro da observação.

O trabalho da pesquisadora ocorreu no período de agosto de 2007 a fevereiro de 2009. Nesse período, a principal estratégia desenvolvida pela gestão para a produção da integralidade era a Assessoria Técnica na Atenção Básica, que foi a experiência analisada. A equipe responsável por essa ação estava lotada no nível central e era composta por trabalhadores da rede municipal (cinco enfermeiros, oito médicos e um odontólogo).

Inicialmente, observaram-se as visitas dos assessores técnicos às unidades de saúde da família (USF) e sua reunião semanal de educação permanente. A partir da observação, foram realizadas entrevistas semi-estruturadas e narrativas escritas. Os critérios de escolha foram: ser integrante da equipe de assessoria técnica, atuar em serviços ligados à assistência direta ao usuário, ou ter relação com a equipe de assessores técnicos. Foram sujeitos da pesquisa dezessete membros da equipe gestora, sendo 14 assessores técnicos e três da equipe diretora central da Secretaria Municipal de Saúde, incluindo a Secretária. Foram observadas 5 USF, uma de cada região urbana. Nelas, além da observação, foram realizadas três entrevistas e duas narrativas escritas.

Neste texto, os ocupantes de cargos formais de gestão são denominados de gestores, ou de equipe gestora; embora os assessores fizessem parte dessa equipe, estão referidos como assessores técnicos ou apenas assessores, e os demais, como trabalhadores. No entanto, vale aqui já explicitar que, como Merhy et al. 1 (p. 24), entendese que "todos os atores governam e ao disputar seus projetos transformam todo o cenário" construído na gestão.

Tomamos como referencial a discussão que Merhy et al. 1 fazem acerca do trabalho em saúde e suas tensões. Tensão entre o trabalho vivo em ato - que ocorre no exato momento da atividade produtiva e criativa, inscrito nas relações entre trabalhador e usuário no volátil momento de seu encontro - e trabalho morto, expresso por meio dos instrumentos, normas e saberes, produzidos anteriormente e disponíveis para ser utilizados. Esse modo de fazer, previamente estruturado, "pode capturar" o trabalho vivo em ato de tal modo, que o trabalhador não exerça sua ação de maneira autônoma e criativa. Essa captura diminui as possibilidades de responder à singularidade de cada encontro e às necessidades de cada usuário. A tensão opera o tempo todo no cotidiano dos serviços, nos vários encontros: dos trabalhadores entre si e deles com os usuários e com gestores.

Outra tensão presente no trabalho em saúde é a constante disputa entre os interesses priva- 
dos de cada trabalhador e os interesses dos usuários, interferindo na definição da organização do processo de trabalho. Então, a produção do cuidado e da integralidade depende "da possibilidade de permeabilizar os espaços institucionais no interior dos serviços de saúde para neles atuar a força instituinte do usuário, mesmo não estando ele fisicamente ali" 1 (p. 31). Merhy et al. 1 (p. 35) indicam a necessidade de enfrentar tais tensões constitutivas do trabalho em saúde cotidianamente, reconhecendo que a "saúde é um território de práticas em permanente estruturação, ontologicamente conflitante conforme os sujeitos colocados em cena".

Este artigo apresenta um recorte dos resultados da pesquisa, discutindo o modo como a regulação assistencial foi organizada, com ênfase no arranjo da assessoria técnica para o apoio à produção do cuidado integral, trazendo para o debate os coletivos organizados e outros dispositivos para a produção da integralidade.

Coletivo entendido como grupo de pessoas organizadas para um fim, que não configura uma unidade, um organismo, mas um dispositivo. Desse modo, ele se propõe como, "produção de encontro, de intercâmbio de provocações, de alteridade" 11 (p. 170). O que os organiza "não é a identidade entre seus membros, mas seu objetivo de produção" 11 (p. 170). Segundo Ceccim 11, coletivos que atuam como dispositivos não requerem homogeneidade, mas capacidade de trabalhar com as diferenças, deixando as prescrições de lado. Dessa forma, não operam modos de produção de cuidado exemplares, mas encontros, ético-politicamente comprometidos com a defesa da vida.

Durante todo o processo foram respeitados os princípios éticos da pesquisa, tendo este estudo sido aprovado pelo Comitê de Ética e Pesquisa do Hospital Universitário Clementino Fraga Filho da Universidade Federal do Rio de Janeiro (UFRJ), sob o no. 549/07. Todos os sujeitos foram convidados a participar, esclarecidos sobre os procedimentos éticos quanto ao sigilo e confidencialidade dos dados, orientados sobre a possibilidade de, a qualquer momento, saírem da pesquisa e, estando de acordo, assinaram o Termo de Consentimento Livre e Esclarecido.

\section{Análise dos resultados}

\section{O contexto municipal}

Londrina, com seus 510.707 habitantes tem uma história expressiva na construção do SUS, por seu pioneirismo. Inicialmente, no desenvolvimento de experiências locais de atenção à saúde e na implementação da gestão municipal. Tem uma rede de serviços de saúde bem estruturada com 52 USF com cobertura, naquele período próxima a $70 \%$ da população, bem como serviços especializados e hospitalares que atendem Londrina e região. No âmbito da atenção especializada, há três centros de especialidades públicos, um municipal, um intermunicipal e outro da Universidade Estadual de Londrina, além de outras clínicas privadas contratadas 12 .

Também Londrina enfrenta a mudança do perfil demográfico e epidemiológico, destacando-se o envelhecimento e a as doenças cardiovasculares como principais causas de morte 13 . As condições crônicas e suas agudizações são importante motivo de atenção nas unidades de saúde, colocando em evidência a fragmentação e desarticulação dos processos de trabalho instituídos nos diferentes serviços 13 . Acrescente-se que os serviços de referência ambulatorial e hospitalar estavam organizados de forma restrita e pontual, muitas vezes direcionados pela capacidade de oferta instalada e não pelas necessidades dos usuários do SUS 14. Como nó crítico para a produção da integralidade na cidade foram tomados, pela equipe gestora, a dificuldade de acesso dos usuários às consultas especializadas e a baixa resolubilidade da atenção básica.

Para esta análise da situação de saúde do município, num primeiro momento, foram realizadas oficinas de planejamento estratégico, utilizando dados epidemiológicos, relatórios das conferências municipais, plano municipal de saúde e a vivência da equipe gestora e dos trabalhadores da rede municipal. Nesse processo, a identificação dos nós críticos foi compartilhada com o conjunto dos trabalhadores.

Foi então proposto o projeto de Regulação da Atenção à Saúde, com o objetivo de aumentar a resolubilidade da atenção prestada nos diversos pontos da rede e integrar as diferentes etapas de atenção. As ações propostas foram: Contratualização dos Serviços de Saúde; Regulação do Acesso à Assistência; Avaliação, Controle e Auditoria sobre os Serviços Prestados e Auditoria Assistencial 13.

A seguir apresentamos os resultados da análise desse processo.

\section{A assessoria técnica na atenção básica - uma ação no cotidiano}

Para construir a regulação do acesso à assistência, a equipe gestora tomou como pressuposto a necessidade de criar espaços de diálogo entre os profissionais dos diferentes pontos da rede de atenção, tomando a educação permanente como ferramenta central para produção de uma 
atenção básica resolutiva. Pretendia-se ampliar a responsabilização e o vínculo dos profissionais em relação ao cuidado prestado e aos usuários, construindo modos para a atenção básica assumir coordenação da atenção à saúde e do fluxo dos usuários nos diversos pontos da rede de atenção. Dentre as estratégias utilizadas destacamos a criação e instituição dos protocolos assistenciais, a gestão da clínica e o apoio à rede produzido pela equipe de assessores técnicos 13 .

Essa equipe foi criada para ativar e apoiar a reorganização do processo de trabalho nas USF, bem como qualificar encaminhamentos e o trânsito dos usuários pelos diversos pontos da rede. Os assessores eram ligados diretamente à Superintendência da Autarquia Municipal de Saúde e seu papel era desencadear mecanismos vivos e ativos para mobilizar o debate e a reflexão dos trabalhadores sobre o processo de cuidado.

A equipe assessora desenvolvia suas ações de apoio com base na regulação da assistência, na gestão da clínica, na vigilância em saúde e na gestão de processos de trabalho. Outros profissionais - médicos, enfermeiros, fisioterapeutas, fonoaudiólogo e profissionais técnico-administrativos do nível central da secretaria - mantinham constante diálogo com os assessores. Desse modo, eram efetivados os encaminhamentos referentes à articulação e à consolidação da rede de cuidados em saúde na área ambulatorial e hospitalar.

Nas USF havia uma relação direta dos trabalhadores com os Assessores Técnicos, que estavam organizados em duplas, compostas por médico e enfermeiro. Cada dupla era responsável por 8 unidades na área urbana e por 12 unidades na área rural. A única odontóloga da equipe assessorava as 17 equipes de saúde bucal do município.

A gestão do cuidado foi compreendida, segundo documentos da secretaria, como produção do cuidado orientado pelas necessidades de saúde do usuário, e a abordagem integral foi construída por meio de criação ativa de espaços de conversa com e entre os trabalhadores da atenção básica e especialidades. Utilizaram, para isso, ferramentas como a gestão da clínica e a auditoria assistencial, implementadas por meio de discussão de casos, protocolos assistenciais e da educação permanente para problematização dos processos de trabalho.

A articulação em rede foi produzida pela utilização de instrumentos mais específicos da regulação e da construção das linhas de cuidado, incluindo padronização das solicitações de procedimentos, protocolos, buscando-se integração e corresponsabilização dos trabalhadores das unidades de saúde nos diferentes pontos da rede. Esse arranjo foi construído por intermédio de encontros, de múltiplas conexões e de intensa rede de conversações.

O trabalho da regulação assistencial iniciouse tomando como objeto as filas de espera para acesso às consultas especializadas. Para melhor compreender as causas desse problema e envolver outros atores, os assessores analisavam, em conjunto com as equipes de saúde da família, a fila de espera por especialidade. Neste artigo apresentaremos como exemplo, a fila de endocrinologia - a primeira a ser enfrentada. Essa especialidade foi escolhida por ser considerada crítica - pela demora e pelos indicadores de morbimortalidade do município.

A fila de espera era uma lista virtual de pessoas encaminhadas pelos profissionais das USF aos serviços especializados. Conforme a disponibilidade mensal de vagas, a lista de espera "rodava”. O critério para inserção na lista e acesso às vagas era cronológico. Constataram: encaminhamentos incompletos e repetidos, usuários que desconheciam o encaminhamento, usuários que já tinham resolvido seu problema de outras formas, casos urgentes perdidos na fila. Enfim, uma fila de espera com informações insuficientes, desatualizadas e "organizadas" de modo a não atender às necessidades do usuário, nem garantir o acesso aos serviços em tempo adequado e oportuno. Foi também constatado que as equipes da atenção básica se desresponsabilizavam do caso ao inscrever o usuário na fila de espera. Ou seja, não havia gestão do processo de referência.

Os usuários foram identificados por nome, registro, data de entrada na fila e se primeira consulta ou retorno. Com base nesses dados, foram agrupados por área de abrangência das USF. Com essas informações, os assessores foram às unidades e iniciaram o levantamento dos prontuários para análise de cada caso. Para tanto, tinham como referência o protocolo clínico e o fluxograma de encaminhamento, previamente elaborados pela equipe de assessoria e um endocrinologista de um dos centros de especialidades. As equipes locais participaram ativamente desse processo, pois conheciam de perto os usuários e traziam para a discussão dos casos as potencialidades e dificuldades do cotidiano. Além da exploração de cada caso, assessores e trabalhadores das unidades problematizaram o tema da responsabilização. Vale dizer, a equipe de referência deveria se ocupar da gestão do "trânsito" e do cuidado do usuário pelos vários itinerários do sistema de saúde.

Foram definidos conjuntamente critérios de priorização e atuou-se a fim de garantir o atendimento às situações efetivamente pertinentes, 
o que gerou satisfação. O especialista participou de todo o movimento, não como consultor, mas como sujeito do processo, que se reconhecia como portador de saberes e práticas, interesses e projetos.

Um dos assessores narra: "Ao mesmo tempo, tínhamos que estudar a 'fila' da endocrinologia, levantar os prontuários, sentar com a equipe, conhecer as pessoas atrás dos prontuários por meio das informações das equipes e tentar, todos juntos, encontrar um caminho que melhorasse a assistência a essas pessoas, reduzindo o tempo de espera por uma consulta com o especialista ou discutindo com a equipe a linha de cuidado" (assessor A).

No caso da endocrinologia, foram analisados 1.529 casos. Desses, 1.059 (68,6\%) poderiam ser acompanhados pelos profissionais da equipe de saúde da família com o apoio técnico da equipe de assessores. Outros 202 usuários (13,2\%) apresentavam obesidade que podia ser acompanhada na USF mediante estratégias específicas para perda de peso em nível ambulatorial. A abordagem à obesidade foi desenvolvida juntamente com os Núcleos de Apoio em Saúde da Família e profissionais da Residência Multiprofissional em Saúde da Família. Somente 6,2\%, isto é, 95 usuários precisavam efetivamente ser encaminhados ao endocrinologista. Os demais 182 casos (11,9\%) não puderam ser analisados por não serem localizados os usuários (por mudança de cidade ou por não haver prontuário no SUS) 15 .

Várias estratégias foram criadas para a efetivação do trabalho em rede. Variaram de acordo com características específicas de cada USF, de cada assessor e da relação entre eles e os trabalhadores das unidades, produzindo-se uma intensa rede de conversações. De um modo geral, sempre que possível, os assessores participavam das reuniões de cada equipe da saúde da família e do conjunto das equipes das USF. Essa presença possibilitou a ampliação do debate acerca da regulação, a compreensão das dificuldades vividas pelas equipes e a construção compartilhada de soluções para o enfrentamento dos problemas. Dessa forma, foi possível apreender as diferenças e conflitos das equipes diante das diversas situações, identificar e inventar novos modos de lidar com o processo do cuidado nos marcos da integralidade. Assim, os assessores foram compondo, com os trabalhadores das unidades, coletivos gestores descentralizados e passaram a desencadear intervenções singulares, sempre articulando e aproximando estes coletivos de outras diretorias da secretaria.

A educação permanente em saúde era utilizada para analisar o trabalho e as práticas, possibilitando identificar problemas, suas causas e produzir novos encaminhamentos. Em reuniões, oficinas e nas discussões de caso eram abordados os problemas de cada semana e elaboradas propostas para atuar ao longo do processo, alterando na prática a verticalidade na tomada de decisão.

Apesar dos resultados, tal operação também gerou desconfortos. Nas conversas do cotidiano observadas havia comentários como "agora eu tenho que ir atrás dos usuários na fila de espera, e tenho que perder tempo com isso, antes era só pôr na fila". Ou seja, apesar de reconhecerem os benefícios do processo para a qualificação do cuidado e de terem maior satisfação em relação a vários aspectos de seu fazer cotidiano, alguns trabalhadores não deixaram de se incomodar com a mudança da lógica na implantação das rotinas e procedimentos.

Estava presente o tensionamento entre os interesses privados (o conforto e a facilidade para os trabalhadores das unidades ao colocar o usuário “na fila”) e a publicização (reorganização do processo de trabalho considerando as necessidades dos usuários). Esse aumento do trabalho decorria da necessidade de preenchimento de impressos, do aumento da quantidade de visitas domiciliares, enfim da gestão do "trânsito" e do cuidado do usuário pelos vários itinerários do sistema de saúde.

Os coordenadores das USF avaliaram este aspecto do trabalho da assessoria como um grande avanço, que contribuiu para o enfrentamento das situações de conflito e colaborou na qualificação da relação entre as unidades e delas com a equipe gestora de nível central 13. Segundo eles, o fácil acesso aos assessores, bem como sua disponibilidade corroboraram para o aumento da segurança das equipes em seu trabalho.

De acordo com a narrativa de um trabalhador de USF: "Quando faltava médico, [o assessor] atendia na unidade ou realizava visita domiciliar, discutia os casos mais complexos com os funcionários (...) colaborava para a resolução de problemas, principalmente quando envolviam outros setores e o nível central. (...) Na minha opinião, em cada região as assessorias trabalham diferente, na unidade percebo que as assessoras realizam atividades parecidas com as que eram realizadas pela coordenação regional, porém de forma mais ampla (caso médico), mais próxima (conhecem a equipe, necessidades, dificuldades) $e$ procuram trabalhar junto" (trabalhador C).

Todos os problemas, as pequenas vitórias e frustrações eram discutidos nas reuniões semanais de educação permanente dos assessores técnicos, quando se analisavam os problemas da prática cotidiana dos participantes e se propunham encaminhamentos. 
Um gestor comenta que: “...foram muito marcantes também as possibilidades que se criavam a todo o momento de refletir no coletivo as situações vivenciadas e também no coletivo definir as ações para enfrentamento dos problemas. A aprendizagem foi ímpar, a lógica da educação permanente em saúde já estava instituída" (gestor M).

A Assessoria Técnica incentivava a utilização, pelas equipes, dos protocolos assistenciais, mas sem desconsiderar a singularidade na produção dos projetos terapêuticos. Assim, esses protocolos foram discutidos com as equipes, num processo de educação permanente em saúde, ou seja, à medida que as necessidades surgiam, valendo-se da prática e do contexto de cada unidade.

Segundo eles, a educação permanente de fato permeava todas as ações dos assessores, considerando que a produção das mudanças se dá sempre num espaço de aprendizagem e afetação múltipla. Portanto, a ação cotidiana dos apoiadores pressupunha a criação de espaços de reflexão da prática dos trabalhadores da atenção básica, em constante diálogo com a atenção especializada e com as demais diretorias da secretaria, sempre que necessário. Dessa forma, segundo relatório 13 , os assessores pretendiam apoiar, conduzir e responsabilizar os trabalhadores pela produção do cuidado nas unidades e tornaram-se dispositivos permanentes para promoção de mudanças na organização do processo de trabalho.

Além disso, o espaço de educação permanente dos próprios assessores, em conjunto com a equipe gestora ampliada, proporcionou a tomada de decisões estratégicas. A equipe gestora ampliada, composta pelas diretorias da Autarquia Municipal de Saúde, e coordenadoras das unidades de saúde, destacou alguns pontos essenciais em relação aos assessores:

- Presença nas unidades com frequência e periodicidade estabelecidas e acordadas com as equipes;

- Acolhimento e busca conjunta de soluções para as demandas trazidas pelos trabalhadores das unidades;

- Capacidade de se solidarizar com a equipe local, sem querer falar por ela (reconhecendo e exercitando a alteridade);

- Capacidade de analisar criticamente o projeto, juntamente com as equipes, adequando-o de acordo com as avaliações realizadas ao longo do processo e superando seus limites;

- Mediação em relação às posições diferentes dos vários atores institucionais.

Participavam também das reuniões semanais de educação permanente em saúde os gestores dos dois centros de especialidades, responsáveis pela maioria dos atendimentos especializados. Sua presença foi importante para assegurar o en- volvimento dos especialistas, para produzir discussões sobre seu processo de trabalho e sobre a relação com os profissionais da atenção básica. Os especialistas foram envolvidos conforme era desencadeada a regulação de sua especialidade.

De acordo com Relatório sobre o Processo de Reorganização do Atendimento na Área de Média Complexidade Ambulatorial no Município de Londrina 16, após esse processo, o tempo de espera para as especialidades reguladas (cardiologia, endocrinologia, neurologia pediátrica, fonosurdez, fisiatria, fisioterapia, mastologia, dermatologia sanitária, saúde auditiva e colposcopia) era no máximo de 30 dias.

Cecílio 17 e Merhy et al. 1, apoiados no reconhecimento da existência do trabalho vivo em ato, que gera espaço de autonomia e liberdade ao trabalhador da saúde, sugerem que os serviços desenvolvam efetivamente processos de gestão pactuados e dirigidos colegiadamente pelo conjunto de seus trabalhadores. Ainda sugerem que esses pactos sejam orientados por contratos centrados na lógica dos usuários, produzindo coletivos implicados com a produção do cuidado e articulados a uma rede regulada pelo Estado.

$\mathrm{O}$ arranjo cuidador foi uma produção em ato, produzido nos encontros, na dinâmica das relações de poderes, na resistência, movimentando, aquecendo, vivendo pequenas lutas diárias. Essas afetações permitiram um "se" colocar em análise todo o tempo e refletir sobre os modos de pensar a organização e seus instituídos. Dessa forma, o cuidado aqui é debatido como resultado de uma complexa trama de atos, de procedimentos, de fluxos, de rotinas, de saberes, todos em disputa e complementaridade, entendendo a integralidade da atenção recebida decorrente da forma como se articulam a prática dos trabalhadores 18 . As redes se produziam nas falas, nos atos e movimentos, aproximando-se da concepção de rede de Kastrup 19 (p. 17), "uma organização que é complexa, aberta, dispersa, sem centro unificador", e que tem como princípio o encontro e a produção de conexões.

Há que se considerar também que nesse arranjo a regulação representou uma intervenção do gestor na ação dos médicos, particularmente em sua relação com os usuários, já que se produziram regras para "controlar", "regular" a caneta do médico. Foram, sim, introduzidos alguns constrangimentos e racionalização na solicitação de exames e no encaminhamento aos especialistas, considerando as necessidades e também a disponibilidade de recursos. Mas essa intervenção foi produzida em diálogo e com a participação desses profissionais e não por meio de mecanismos de controle. Para tanto, também foram utilizadas ferramentas da educação per- 
manente. Além disso, a regulação foi trabalhada de modo concomitante com a produção ativa de aproximação entre distintos lugares da rede, com a lógica do matriciamento e da reflexão compartilhada sobre as diversas situações.

Vale ressaltar a coerência da operação com os pressupostos do projeto. O gestor propunha que os assessores tivessem "ação direta na assistência aos pacientes em conjunto com os médicos das unidades" 15 (p. 2).

Portanto, era imprescindível estabelecer diálogo na perspectiva clínica, com os profissionais médicos das USF e, ao mesmo tempo, com especialistas que atuavam nas clínicas de especialidades, tanto públicas quanto privadas. E tal diálogo foi produzido na perspectiva de quem tinha já alguma vivência na área, pois ter experiência na clínica e habilidades técnicas para poder problematizar condutas médicas foram atributos indispensáveis dos assessores no arranjo proposto.

A fala do gestor evidencia que " a presença do especialista discutindo casos com a equipe na USF. Em especial uma situação ocorrida em que o especialista juntamente com a equipe da saúde da família fez uma visita domiciliar a um paciente que vivia isolado. Foi ótimo aproximar especialistas da equipe de atenção básica. É imprescindivel a figura de um apoiador no cotidiano de uma unidade de saúde" (gestor S).

Assim, o matriciamento foi entendido como uma ação de agregar e combinar diferentes saberes para enfrentar a complexidade dos problemas de saúde, produzindo conhecimento mútuo e trocas, reconhecendo os saberes produzidos no cotidiano e produzindo relações de cooperação. Cotidiano é o tempo em que acontece o encontro do trabalhador com o usuário, momento do trabalho vivo em ato, é o tempo-lugar das disputas, tempo-espaço de luta, de exercício de poder. É também o terreno da produção e da cristalização dos modelos de atenção à saúde, e onde podem se configurar "novos espaços de ação $e$ novos sujeitos coletivos, bases para modificar o sentido das ações de saúde, em direção ao campo das necessidades dos usuários finais” 20 (p. 306).

Em varias situações, como em relação à obesidade, realizou-se um trabalho multidisciplinar e multiprofissional, necessário para o manejo dessa condição. Nestas situações, havia a participação de profissionais dos Núcleos de Apoio à Saúde da Família (educação física, psicologia, fisioterapia, odontologia, farmácia e nutrição).

O arranjo, como produzido e operado, interferiu sobre a clínica e sobre o cuidado no cotidiano, implicou a produção de novos atos de saúde e de novos modos de gestão da clínica, do cuidado e do cotidiano.

\section{Discussão e considerações finais}

A construção do projeto deu-se ao mesmo tempo em que se constituiu o coletivo com liberdade de criar. O dispositivo produziu rupturas, desencadeou novas práticas de gestão, interrogou a prática dos trabalhadores da saúde e possibilitou novas formas de produção do cuidado. Aos poucos, quebrou-se a distância entre o gestor "do nível central” e os trabalhadores das unidades. Mas neste processo foi necessário lidar com limitações, dificuldades e complexidades na relação com os trabalhadores e com as equipes. Efetuouse no cotidiano o debate da integralidade e a coresponsabilização das equipes pelo trânsito dos usuários na rede mediante a problematização do processo de trabalho clínico. Desse modo, o trabalho médico (e da equipe) foi questionado e reorientado para a produção do cuidado. A integralidade foi considerada em sua polissemia, produzida pelos sujeitos do cotidiano, com suas vivências e saberes, incluindo o usuário com seu desejo de ter acesso aos serviços de saúde, de ser bem tratado e com qualidade 21 .

Este estudo enfocou o modo como foi organizado e se desenvolveu o arranjo da assessoria técnica para efetuar a regulação assistencial. Foi um estudo de abordagem qualitativa, que não tem como objetivo quantificar, mas contribuir para a compreensão dos processos de produção do cuidado, sem propor generalizações. É uma demonstração de que é possível racionalizar recursos com base na qualificação dos processos e das relações 22 .

Este trabalho esteve em consonância com estudo sobre a regulação da atenção à saúde em Belo Horizonte 5, em que os autores indicam que a garantia da integralidade implica operar mudanças na produção do cuidado, com base na rede de atenção em saúde em todos os níveis assistenciais. Acrescentam que a integralidade não é garantida apenas com a organização dos recursos disponíveis, mas com a própria prática dos trabalhadores e com fluxos que garantam o acesso do usuário aos serviços de que necessitam.

Os resultados de outro estudo realizado por Rosa et al. 23 sobre a integralidade da atenção às doenças cardiovasculares e diabetes mellitus, no Estado de São Paulo, destacaram a regulação como alternativa para assegurar redes assistenciais resolutivas. Apontaram, no entanto, entraves do processo de produção da integralidade e destacaram a insuficiência dos mecanismos formais de referenciamento bem como dos instrumentos para o seu acompanhamento. Indicam ainda que "a inexistência de protocolos de encaminhamento - na Atenção Básica - e o perfil 
dos médicos podem levar ao aumento da demanda por serviços de média e alta complexidade" 23 (p. 165).

Tais resultados vão ao encontro das discussões deste trabalho em relação à necessidade de conformar redes de cuidado e sua regulação com mecanismos que possibilitem encontros, conversações na perspectiva clínica e negociações, num processo extremamente singular e mutável, respeitando as possibilidades das equipes e as necessidades dos usuários. Logo, para a garantia oportuna do acesso aos diversos serviços de saúde, de forma equânime e de acordo com a necessidade do usuário, não é suficiente ter em conta apenas os aspectos técnicos dos protocolos assistenciais. Embora sejam necessários, é preciso também endereçar as relações entre os envolvidos no trabalho em saúde nos diversos pontos da rede de atenção. Assumem, neste momento relevância, as tecnologias leves, utilizadas para estabelecer relações, que podem ser de vínculo, responsabilização, escuta qualificada, cooperação 17. A efetuação do acesso e a produção do cuidado integral a determinado usuário em determinado serviço de saúde são realizadas pelo conjunto dos trabalhadores de saúde em ato, nas tensões e disputas presentes na gestão do cotidiano.

Estudos realizados por Giovanella 7 e por Nolte $\&$ Mckee 6 reforçam a necessidade de arranjos organizacionais em rede baseados em tomada de decisão coletiva, na cooperação e no diálogo para que seja possível superar as barreiras existentes para a coordenação do cuidado em saúde e a integralidade.

Feuerwerker \& Merhy 4 apontam a necessária produção de linhas de cuidado que atravessem a atenção básica, especializada, ambulatorial e hospitalar. Para tanto, novas modalidades de apoio e articulação entre trabalhadores e serviços precisam ser inventadas, levando em conta a necessidade de atender à intensificação do cuidado requerido pelos usuários em suas distintas situações de vida. Modalidades que possibilitem "uma análise crítica permanente sobre o proces- so de trabalho, bem como a constante renovação de pactos" 4 (p. 181) entre trabalhadores de uma mesma equipe e entre diferentes equipamentos de saúde, em arranjos de responsabilidade compartilhada pelo cuidado.

Escorel et al. 24 afirmam que os mecanismos de comunicação com troca de informações entre profissionais dos diversos serviços de saúde são essenciais para os trabalhadores da atenção básica exercerem a coordenação e garantirem a continuidade do cuidado aos usuários. Todavia, os mecanismos existentes à época da pesquisa realizada em municípios brasileiros eram insuficientes de acordo com a maioria dos gestores municipais.

A análise dos resultados deste estudo demonstra que, para avançar na conquista da integralidade, há necessidade de arranjos que articulem a regulação com a efetiva produção da rede de cuidados. Isto é, dispositivos que atuem na articulação e coordenação da rede que está em constante movimento e transformação.

Ao atuar sobre as tensões presentes no cotidiano do trabalho em saúde, evidencia-se que as tecnologias já disponíveis são insuficientes para articular a gestão do sistema em direção à produção do cuidado centrado na necessidade do cidadão. Elas precisam ser enriquecidas em ato pelos saberes e tecnologias produzidos em encontros deliberados, em movimentos ativos da gestão para apoiar a produção do cuidado. Conseqüentemente, indicamos que a produção da integralidade, princípio do SUS, exige que a gestão tome a produção do cuidado e o trabalho em saúde como objetos de ação, como política de governo. Não basta montar sistemas de informação, fluxogramas, protocolos e normas. É necessário efetuá-los no cotidiano, na interação entre os diversos serviços, com aqueles que ali estão presentes, gestores, trabalhadores e usuários. Nos encontros e nas redes de conversação é que acontece a produção do trabalho em saúde e se efetuam os princípios de valorização da vida e da integralidade, centrais para a consolidação do SUS. 


\section{Resumo}

A produção do cuidado integral e a eqüidade são desafios na gestão do SUS. Analisou-se um arranjo da gestão municipal para a produção do cuidado implantado por meio da regulação assistencial com o objetivo de enriquecer as reflexões sobre o tema. Foi realizado estudo de caso em que os dados foram obtidos mediante observação, entrevistas, narrativas escritas e análise documental. Os sujeitos da pesquisa foram gestores e trabalhadores da Secretaria de Saúde. Como resultado destaca-se um conceito de rede viva, produzida ativamente pela construção de relações entre os trabalhadores dos diferentes serviços. Destacam-se as ações de apoio ao cuidado produzido pelas equipes, com base na regulação assistencial e na gestão dos processos de trabalho. Os resultados foram expressivos na qualificação do cuidado, na redução das filas às especialidades e na satisfação dos profissionais. Como marca do processo, evidencia-se a necessidade de enriquecer, em ato, os saberes técnicos com as tecnologias leves e os saberes produzidos em movimentos ativos da gestão para a produção do cuidado.

Gestão em Saúde; Assistência Integral à Saúde; Saúde da Família

\section{Colaboradores}

Todos os autores contribuíram na concepção, redação e análise dos dados do artigo.

\section{Referências}

1. Merhy EE, Magalhães Júnior HM, Rimoli J, Franco TB, Bueno WS, organizadores. O trabalho em saúde: olhando e experienciando o SUS no cotidiano. São Paulo: Editora Hucitec; 2003.

2. Santos NR. Desenvolvimento do SUS, rumos estratégicos e estratégias para visualização dos rumos. Ciênc Saúde Coletiva 2007; 12:429-35.

3. Word Health Organization. Innovative care for chronic conditions: building blocks for action. Geneva: World Health Organization; 2002.

4. Feuerwerker LCM, Merhy EE. A contribuição da atenção domiciliar para a configuração de redes substitutivas de saúde: desinstitucionalização e transformação de práticas. Rev Panam Salud Pública 2008; 24:180-8.
5. Franco T, Magalhães Júnior HM. Integralidade na assistência à saúde: a organização das linhas do cuidado. In: Merhy EE, Magalhães Júnior HM, Rimoli J, Franco TB, Bueno WS, organizadores. O trabalho em saúde: olhando e experienciando o SUS no cotidiano. São Paulo: Editora Hucitec; 2003. p. 125-32.

6. Nolte E, Mackee M. Caring for people with chronic conditions: a health system perspective. New York: Open University Press/McGraw Hill Education; 2008.

7. Giovanella L. A atenção primária à saúde nos países da União Européia: configurações e reformas organizacionais na década de 1990. Cad Saúde Pública 2006; 22:951-63. 
8. Epping-Jordan JE, Pruitt SD, Bengoa R, Wagner EH. Improving the quality of health care for chronic conditions. Qual Saf Health Care 2004; 13:299-305.

9. Iriart C, Merhy EE, Waitzkin H. La atención gerenciada en América Latina. Transnacionalización del sector salud en el contexto de la reforma. Cad Saúde Pública 2000; 16:95-105.

10. Ministério da Saúde. Diretrizes operacionais dos pactos pela vida, em defesa do SUS e de gestão. http://www.saude.caop.mp.pr.gov.br/arquivos/Fi le/volume1.pdf (acessado em 22/Fev/2010).

11. Ceccim RB. Onde se lê "recursos humanos em saúde" leia-se "coletivos organizados de produção da saúde". In: Pinheiro R, Mattos R, organizadores. Construção social da demanda: direito à saúde, trabalho em equipe, participação e espaços públicos. Rio de Janeiro: ABRASCO; 2005. p. 161-80.

12. Prefeitura Municipal de Londrina. Plano municipal de saúde para o biênio 2004-2005. Londrina: Autarquia do Serviço Municipal de Saúde; 2004.

13. Autarquia Municipal de Saúde, Prefeitura Municipal de Londrina. Relatório de trabalho da assessoria técnica na atenção básica - de junho de 2007 a dezembro de 2008. Londrina: Autarquia Municipal de Saúde; 2009.

14. Ramos MLR, organizadora. 6a mostra de experiências da Autarquia Municipal de Saúde. Londrina: Autarquia Municipal de Saúde; 2008.

15. Autarquia Municipal de Saúde, Prefeitura Municipal de Londrina. Equipe de reguladores aumenta a qualidade do atendimento nas unidades básicas de saúde. Boletim Informativo da Saúde 2007; 12:2.
16. Autarquia Municipal de Saúde, Prefeitura Municipal de Londrina. Relatório sobre o processo de reorganização do atendimento na área de média complexidade ambulatorial no município de Londrina. Londrina: Autarquia Municipal de Saúde; 2008.

17. Cecílio LCO, organizador. Inventando a mudança na saúde. São Paulo: Editora Hucitec; 1994.

18. Merhy EE, Cecílio LCO. O singular processo de coordenação dos hospitais. Saúde Debate 2003; 64:110-22.

19. Kastrup V. A psicologia na rede e novos intercessores. In: Fonseca TMG, Francisco DJ, organizadores. Formas de ser e habitar a contemporaneidade. Porto Alegre: Editora da UFRGS; 2000. p. 13-26.

20. Merhy EE. Saúde: a cartografia do trabalho vivo. São Paulo: Editora Hucitec; 2002.

21. Mattos RA. A integralidade na prática (ou sobre a prática da integralidade). Cad Saúde Pública 2004; 20:1411-6.

22. Conill EM. Ensaio histórico-conceitual sobre a Atenção Primária à Saúde: desafios para a organização de serviços básicos e da Estratégia Saúde da Família em centros urbanos no Brasil. Cad Saúde Pública 2008; 24 Suppl 1:S7-27.

23. Rosa TEC, Bersusa AAS, Mondini L, Saldiva SRDM, Nascimento PR, Venancio SI. Integralidade da atenção às doenças cardiovasculares e diabetes mellitus: o papel da regionalização do Sistema Único de Saúde no Estado de São Paulo. Rev Bras Epidemiol 2009; 12:158-71.

24. Escorel S. O Programa de Saúde da Família e a construção de um novo modelo para a atenção básica no Brasil. Rev Panam Salud Pública 2007; 21:164-76

Recebido em 12/Mai/2010

Versão final reapresentada em 09/Set/2010 Aprovado em 15/Out/2010 